Mangelhafte Versorgung in Ärztenetzwerken Zum erwähnten Beitrag [1] kann ich Herrn Professor Baur nur gratulieren. Nicht nur ist die zur Eindämmung der Gesundheitskosten gedachte Bevorzugung von Ärztenetzwerken ein problematischer Vorschlag, der zu mangelhafter Versorgung führen kann, vielmehr wird ein qualitativ hochstehendes Gesundheitsversorgungssystem ernsthaft gefährdet. Das KVG der neunziger Jahre sollte eine Mehrklassenmedizin verhindern, indem die Politiker, nicht wir Ärzte, einen umfangreichen Grundleistungskatalog gestaltet haben, nun sollen die Kostenfolgen naive Ärzte ausbaden, indem sie sogenannte Budgetverantwortung tragen. Damit wird durch die Hintertür genau jene Mehrklassenmedizin eingeführt, die man verhindern wollte. Wer es sich leisten kann, dazu rechne ich sicher auch Herrn Bundesrat Couchepin, begibt sich kaum in ein Ärztenetzwerk, um Prämien zu sparen. So behält die alte Weisheit ihren wahren Kern: Es ist besser reich, schön und gesund zu sein, als arm, hässlich und krank. Und wenn schon krank, dann sind die Überlebenschancen, wie Statistiken eindrucksvoll belegen, mit einem gut ausgestatteten Portefeuille wesentlich besser.

Wer daran verdient, Leistungen zu unterlassen, wird Leistungen unterlassen. Wer umgekehrt daran verdient, Leistungen zu erbringen, wird möglicherweise zu viele Leistungen erbringen. Ich jedenfalls möchte nicht, dass auf meinem Buckel und auf Kosten meiner Gesundheit Kosten eingespart werden.

Wie absurd das System mit Budget- statt Patientenverantwortung ist, lässt sich mit einem Vergleich illustrieren: Sie haben einen Wasserschaden in der Wohnung und bestellen den Sanitärinstallateur. Er lehnt den Auftrag ab, weil er mit dem Verband Schweizer Hausratversicherer einen Vertrag abgeschlossen hat, der ihn an eingesparten Prämiengeldern mitverdienen lässt. Wir müssen endlich aufhören, mit untauglichen Mitteln wie der Abschaffung des Kontrahierungszwanges die Kostenfrage anzugehen. Vielmehr müssen wir der Schweizer Bevölkerung die Frage vorlegen, ob sie die Fortschritte der Medizin wünscht und wieviel dieser Fortschritt kosten darf. Und schliesslich ist auch jeder einzelne in die Pflicht zu nehmen. Wer seine Gesundheit fahrlässig aufs Spiel setzt, soll zahlen.
Zum Beispiel würde eine Lenkungsabgabe von bescheidenen 10 Franken pro Zigarettenpack Wunder wirken.

Dr. med. Daniel Schlossberg, Zürich

1 Baur H-R. Mangelhafte Versorgung in Ärztenetzwerken. NZZ, 26. Mai 2004.

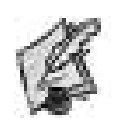

\section{Finanzierung der Belegarztpraxis}

Die Schweizerische Ärztezeitung publizierte einen Bericht der Helsana zur Finanzierung der Belegarztpraxis [1]. Darin spiegeln sich die Überlegungen der Kassenseite zum neuausgearbeiteten Belegarzttarif für Spitalaktivitäten von Belegärzten zu Lasten der sozialen Grundversicherung.

Der TARMED zeugt von einem, in der Zwischenzeit allseits anerkannten, diskriminierenden Verständnismangel der Versicherer bezüglich betriebswirtschaftlicher Belange einer Belegarztpraxis. Die Vorstellungen von Kassenseite sind geradezu beispielhaft zusammengefasst im obgenannten Artikel der Helsana. Dabei werden die üblichen Fehlinterpretationen sehr schön auf den Punkt gebracht: «Die während der Tätigkeit des Belegarztes am Spital anfallenden Praxiskosten könnten auch durch andere Quellen finanziert werden, indem beispielsweise durch geeignete Zusammenarbeitsformen unter den Belegärzten die Praxis dennoch besetzt ist». Dabei wird das arztnahe TL des Belegarzttarifs ganz offensichtlich mit den Praxisinfrastrukturkosten für die ganze Praxis verwechselt. Letztere sind jedoch, beruhend auf Normwerten, in der Tat bereits im TARMED integriert, und zwar eben für den Praxisoperationssaal und nicht für die belegärztliche Tätigkeit am Spital. Dass die Infrastrukturkosten mit einer Auslastung von neun Stunden während sieben Tagen eingerechnet sind, während der Praxisoperationssaal eines Belegarztes neben Spitaltätigkeit und Sprechstundentätigkeit nur an zwei bis drei Halbtagen in Betrieb sein kann, da selbst bei Zusammenschluss mehrerer Belegärzte die obgenannte, am Schreibtisch ausgedachte, traumhafte Idealauslastung nie erreicht werden kann und dass der Belegarzt schlussendlich nur 60\% dieses theoretisch errechneten Tiefstwertes erhält, entspricht einer ersten betriebswirtschaftlichen Fehlleistung des TARMED mit Benachteiligung des Be- 
legarztes, hat aber mit dem Belegarzttarif nichts zu tun. Ebenso lässt sich kein Zusammenhang herstellen mit dem Praxisumsatz, schon gar nicht mit Schätzungen dazu von seiten der Helsana, oder mit der Art und Weise, wie der Belegarzttarif bei stationären Eingriffen in die Spitalpauschale einfliesst.

Das arztnahe TL, um das es im Belegarzttarif geht, scheinen die Kassen ganz offensichtlich gar nicht zu kennen, wie der vorliegende Artikel einmal mehr beweist. Nach deren Vorstellungen schweben die Patienten wie von selbst, exakt zum richtigen Zeitpunkt, pfannenfertig vorbereitet, mitsamt Kostengutsprache der Kasse, auf den Operationstisch des Spitals, wo sie vom Belegarzt nur noch operiert werden müssen. Der heutzutage immer gigantischer werdende administrative Aufwand vor und nach einem operativen Eingriff, die Absprache mit Patient, Operationssaal, Anästhesie, Assistenz, Abteilung usw. und was die sonstigen Voraussetzungen dazu sind, wird bei einem Belegarzt, im Unterschied zu einem Spitalarzt, von der Belegarztpraxis erbracht. Das arztnahe TL hat demnach nichts damit zu tun, ob die Praxis genau während der Operationszeit am Spital offen oder geschlossen ist oder ob die Praxisräumlichkeiten zu diesem Zeitpunkt von einem anderen Belegarzt genutzt werden oder nicht. Einfach ausgedrückt berücksichtigt der Belegarzttarif, welche zusätzlichen Kosten einem Belegarzt, im Vergleich zu einem Spitalarzt, im Zusammenhang mit einem operativen Eingriff am Spital entstehen.

\section{Dr. med. R. Schmoker, Bern}

1 Gyger P, Helfer G, Achermann R. Finanzierung der Belegarztpraxis. Schweiz Ärztezeitung 2004;85(36):1899-1901.

Qualitätssicherung im Röntgen - noch einmal anders ... oder zur klinisch epidemiologischen Evidenz oder wieviel Sicherheitsluxus dürfen wir uns auf Kosten von was und von wem leisten?

Die Replik von Kollege Kuhn hatte mich gefreut [1]. Für mich hatte der Artikel von Herrn Gschwind [2] eigentlich nur Kopfschütteln provoziert. Meine Erfahrungen waren nun wirklich völlig anders. Bei einer Praxisvertretung als Medizinstudent etwa 1970 hatte ich einmal einen Ölfleck unter der Röntgenröhre festgestellt und wusste vom Studium, dass die Röhre tatsächlich auch Öl zum Kühlen braucht. Damals war man sich noch gewohnt, nachzuschauen, wenn ein Apparat nicht funktionierte. Ich fand ein kleines Loch wie bei einem Veloschlauch infolge eines Nagels im schwarzen Gummi des Ölbehälters und behandelte dieses Loch auch genau gleich wie ein Loch beim Fahrradschlauch. Die Sache blieb dicht. Der Röntgenapparat wurde weiter benutzt und beim Abschluss der Ferienvertretung wurde dies dem rückkehrenden Arzt gemeldet. Er soll doch so gut sein und diesen Flick gelegentlich fachmännisch nachkontrollieren lassen. - Heute würde ich mir natürlich nie mehr zutrauen, eine solche Reparatur selbst vorzunehmen!

Ich habe meine eigene Röntgenanlage 1979 installiert und sie wurde seither immer wieder kontrolliert, es gab auch gelegentlich kleine Lampen, die ihren Dienst versagten, aber nie kam ich in die Lage, notfallmässig den Röntgentechniker herzurufen. Seit nun 25 Jahren kommt auch immer der gleiche Techniker, stets in Kombination mit Besuchen bei Kollegen in Nachbardörfern mit gleichen Apparaten. Es gab gelegentlich kleinere Mängel, welche problemlos behoben werden konnten, wie beispielsweise eine zu schwache Birne im Negatoskop, was die Qualität der Röntgenbilder ja bekanntlich sehr einschränkt. Daneben neue Kassetten (Folien), entsprechende Anpassungen, empfindlichere Filme und neue günstigere Dunkelkammerleuchten, also viele Details auch hinsichtlich Strahlenschutz.

Vor einem Monat bekam ich die offizielle Erlaubnis, weitere 10 Jahre Röntgenbilder mit dieser Anlage zu machen, allerdings unter der Voraussetzung der regelmässigen Kontrollen, wie wir das immer gemacht haben und dem Nachweis des Sachverstandes für Strahlenschutz. Ich hatte dem BAG Abteilung Strahlenschutz klar begründet, weshalb meines Erachtens aufgrund meiner offensichtlich seltenen Benützung der Röntgenanlage ein Nachweis des Sachverstandes in Strahlenschutz nicht nötig sei, weil die Gesamtheit meiner pro Jahr applizierten Strahlendosis am Patienten weit unter der natürlichen Strahlenexposition und um ein vielfaches unter einer einzigen CT-Untersuchung des Abdomens liegt.

Leider ist das Bundesamt für Gesundheit Abteilung Strahlenschutz nicht in der Lage, solche sachliche Fragen konkret zu beantworten, ihr Sachverstand beschränkt sich auf juristische Vorstellungen.

Es gibt in der Schweiz nachweislich seit Jahren keine Strahlenschäden beim nicht dosisintensiven Röntgen in der Praxis. Deterministische Effekte müssten geradezu mutwillig erzeugt wer- 
den. Alle Strahlenschäden beruhen auf Schätzungen stochastischer Effekte. Diese lassen mit Fug und Recht sehr viele Zweifel offen, insbesondere im Hinblick auf die um einige Potenzen höhere Strahlenbelastung in der Nachkriegszeit (Durchleuchten der Füsse beim Schumacher, routinemässiges Durchleuchten aller Spitaleintritte, auch Kinder zur Tonsillektomie, Schirmbilder, usw. - all dies speziell bei Kindern und Jugendlichen!) Irgend etwas müsste hier doch auch vom stochastischen Phänomen erfassbar sein! Wenn nicht, dann muss man sich fragen, ob man die zur Verfügung stehenden Mittel epidemiologisch nicht sinnvoller einsetzt. Dabei geht es nicht nur um Stellen von Experten und Kontrolleuren, sondern auch um die verursachten indirekten Kosten wie z.B. Prüfungen des Sachverstandes von Ärzten, hier speziell um den Verlust der Glaubwürdigkeit, also um ideelle Werte! Für die Verhinderung von Masern-, RötelnHepatitisvireninfektionen kümmert sich kein Jurist, im Gegenteil! Impfungen werden in der Schweiz juristisch behindert statt gefördert! Das Recht des Individuums auf eine Krankheit wird höher eingeschätzt als die Verbreitung einer gelegentlich tödlich verlaufenden Krankheit (z.B. Masern). Wenigstens bei der Tuberkulose scheint das Epidemiegesetz noch einigermassen zu funktionieren.

Viel wichtiger aber als diese Strahlenschutzbelastungsberechnungen sind in der Tat ihre Kosten. In der Epidemiologie gilt beispielsweise hier ein guter Grundsatz: wieviel kostet die Prophylaxe für ein verhütetes verlorenes Lebensjahr?

Wenn wir dies gesamtheitlich mitberücksichtigen, dann wäre wohl die effektivste und billigste Prophylaxe die Jodprophylaxe über das Kochsalz. So nebenbei schützt eine optimale Jodprophylaxe auch bei einem radioaktiven Fallout, wo relativ viel radioaktives Jod entsteht und entsprechend proportional mit dem Jod in der Nahrung aufgenommen wird. Die Frauen und die Bäuerinnen speziell zeigen mindestens in Deutschland eine Jodunterversorgung, sie wären deshalb entsprechend vermehrt strahlengefährdet. Wie weit dies in der Tschernobylregion der Fall war, weiss ich nicht. Kropf und Kretinismus war bis zur Einführung der Kochsalziodierung immerhin der häufigste Grund einer Militärdienstuntauglichkeit.

Auch Impfungen haben eine weit grössere messbare Evidenz. Der weltweit häufigste Krebs, das Hepatom, lässt sich mittels der Hepatitis-B-Impfung weitgehend verhindern, ebenso die Virusübertragung Mutter-Kind als positiver Effekt über Generationen. Schwere Missbildungen mit Rötelnimpfungen sind billig vermeidbar.

\section{Schutz des Personals}

Offensichtlich werden nicht nur in unseren beiden Praxen keine Dosisüberschreitungen beim personellen Dosimeter in den letzten Jahrzehnten mehr festgestellt (auch bei mir im Gegensatz zu früher, als ich noch im Spital arbeitete)! Dies ist ein eindeutiges Zeichen, dass zuviel und somit unnötig gemessen wird. In dieser Situation könnte durchaus im Strahlenschutz wesentlich gespart werden, speziell bei der individuellen Dosimetrie, dies speziell in Praxen, wo kein dosisintensives Röntgen betrieben wird. Es ist schwer vorstellbar, wie eine Arztgehilfin oder ein Arzt mit multiplen Schikanen gesichert hinter einer Bleiwand pro Jahr $25 \mathrm{mSv}$ erhalten könnte!

Sicher werden also zu viele Personen zu häufig dosimetriert. Eine Dosimetrie alle 6 bis 12 Monate beim nichtdosisintensiven Röntgen in der Praxis ist deshalb mehr als nur genügend. Dies würde bereits erhebliche Kosten einsparen!

Vermutlich aber werden die Strahlenschützer stur an ihrem bisherigen Konzept festhalten und eher noch weitere Massnahmen fordern! Ich bin gespannt, ob ich wenigstens darauf vom BAG erstmals eine klare biologisch und nicht juristisch begründete Antwort erhalte, auf meine bisherigen Briefe habe ich nie eine Antwort mit einem sachlichen Bezug erhalten.

Auch in der Präventivmedizin ist es wichtig, die vorhandenen finanziellen Mittel sinnvoll einzusetzen. Dabei müssen auch im Strahlenschutz alle verursachten Kosten addiert werden, also vom Zeitaufwand des Arztes und seines Personals, die Kosten der baulichen Massnahmen, aller Kontrollen apparativer Art und die Kosten der diese Massnahmen prüfenden Beamten, Bewilligungsgebühren und schliesslich auch noch die Dosimetrien...! All dies muss ja letztlich jemand auch bezahlen, in der medizinischen Radiologie eindeutig der Patient über die Krankenkassenprämien! Wenn im Gesundheitswesen gespart werden will, ist es notwendig, auch fragwürdige Strahlenschutzmassnahmen zu hinterfragen!

Dr. med. Markus Gassner-Bachmann, Grabs

1 Kuhn Hp. Qualitätssicherung im Röntgen - noch einmal anders. Schweiz Ärztezeitung 2004; 85(35):1802.

2 Gschwind H. Qualitätssicherung im Röntgen einmal anders ... oder was bringt den Röntgenfirmen die Qualitätssicherung? Schweiz Ärztezeitung 2004;85(29/30):1554-5. 